\title{
INDUCCIÓN DE RESISTENCIA A Mycosphaerella fijiensis MORELET Y SU RELACIÓN CON EL RENDIMIENTO DE PLANTAS DE BANANO (Musa AAA) CV. WILLIAMS
}

\section{INDUCTION OF RESISTANCE TO Mycosphaerella fijiensis MORELET AND ITS RELATIONSHIP WITH YIELD OF BANANA PLANTS (Musa AAA) CV. WILLIAMS}

\author{
Pedro Isaías Terrero Yepez ${ }^{1,2}$, Sofia Lorena Peñaherrera Villafuerte ${ }^{3}$, Antonio Javier Bustamante Gonzales ${ }^{4}$, Galo Alexander Cedeño \\ García $^{5}$, Ronel Fernando Solórzano Alcívar ${ }^{6}$, George Alexander Cedeño García ${ }^{7}$ \\ ${ }^{1}$ Instituto de Posgrado de la Universidad Técnica de Manabí, Av. José María Urbina, Portoviejo, Manabí,Ecuador \\ ${ }^{2}$ Agencia de Regulación y Control Fito y Zoosanitario - AGROCALIDAD, Guayaquil, Guayas, Ecuador \\ ${ }^{3}$ Estación Experimental Tropical Pichilingue, Instituto Nacional de Investigaciones Agropecuarias, Departamento de Protección Vegetal km 5 vía \\ Quevedo-El Empalme, Cantón Mocache, Los Ríos, Ecuador \\ ${ }^{4}$ Estación Experimental Tropical Pichilingue, Instituto Nacional de Investigaciones Agropecuarias, Programa Nacional de Banano, Plátano y otras \\ Musáceas, km 5 vía Quevedo-El Empalme, Cantón Mocache, Los Ríos, Ecuador \\ ${ }^{5}$ Carrera de Ingeniería Agrícola, Escuela Superior Politécnica Agropecuaria de Manabí Manuel Félix López, Campus Politécnico El Limón, sector El \\ Gramal, Calceta, Manabí, Ecuador \\ ${ }^{6}$ Ministerio de Agricultura y Ganadería - MAG, Quito, Pichincha, Ecuador \\ ${ }^{7}$ Departamento de Agronomía, Facultad de Ingeniería Agronómica, Universidad Técnica de Manabí, km 13 1⁄2 vía Portoviejo - Santa Ana, Santa Ana, \\ Manabí, Ecuador \\ Email: correo@insitucional.com
}

\section{Información del artículo}

Tipo de artículo: Artículo original

Recibido: 05/08/2020

Aceptado:

01/12/2020

Licencia: CC BY-NC-SA 4.0

Revista ESPAMCIENCIA 11(2):80-87

DOI: https://doi.org/10.51 260/revista_espamci encia.v11i2.230

\section{Resumen}

El banano es ampliamente sembrado en el Ecuador, siendo el producto agrícola más exportado, sin embargo, la producción de la fruta se ve reducida por enfermedades fitosanitarias como la sigatoka negra. Su modo de control principalmente incluye fungicidas de síntesis química que contribuyen a la contaminación del medio ambiente. Por esta razón, se realiza el presente experimento en la zona central del litoral ecuatoriano, cuyo objetivo fue evaluar a nivel de campo, el efecto de sustancias inductoras de resistencia frente a la evolución de la enfermedad de la sigatoka negra en las épocas seca y lluviosa. El experimento se planteó bajo un diseño de bloques aleatorizado, de 5 tratamientos y 4 bloques. Los tratamientos estudiados fueron T1: ácido jasmónico, T2: ácido salicílico, T3: extracto de carophilaceas, más dos testigos, químico (T4) y absoluto (T5). Se midió el índice de infección, área foliar y hojas funcionales, tanto en la floración como a la cosecha. Adicionalmente se evaluó también el peso neto del racimo. Ácido jasmónico y salicílico, mostraron efecto sobre el índice de infección, en las dos épocas de producción estudiadas. En cuanto al peso neto del racimo, ácido salicílico, registró el mejor promedio, en comparación con los otros tratamientos. Finalmente, con este experimento se demostró que estos inductores de resistencia pueden ser implementados en programas de manejo de la enfermedad, así como también para aumentar el rendimiento del cultivo, reduciendo de esta manera el uso de productos químicos cuyo uso indiscriminado crea resistencia y contaminación ambiental.

Palabras clave: Sigatoka negra, inductores de resistencia, ácido jasmónico, ácido salicílico, extracto de carophilaceas

\begin{abstract}
Bananas are widely grown in Ecuador, being the most exported crop, however, fruit production is reduced by phytosanitary diseases such as black Sigatoka. Management of this disease includes mostly chemical synthesis fungicides that contribute to environmental pollution. For this reason, this experiment was carried out in the central zone of the ecuadorian coastal region, to evaluate at field level the effect of inducing resistance substances against the evolution of "black Sigatoka" in the dry and rainy seasons. The experiment was deployed in a complete randomized block design, with five treatments and 4 blocks. The treatments studied were T1: Jasmonic acid, T2: Salicylic acid, T3: Carophilaceas extract, plus chemical (T4) and absolute (T5) controls. The infection rate, leaf area and functional leaves were measured at both, flowering and harvest stages. Additionally, net bunch weight was also evaluated. Jasmonic and salicylic acid showed a negative effect on the infection rate in both production periods. In terms of net bunch weight, Salicylic Acid registered the best average, compared to other treatments. Finally, this experiment showed that these resistance inducers can be implemented in disease management programs, as well as to increase crop yield, thus reducing the use of chemical products whose indiscriminate use creates resistance and environmental contamination.
\end{abstract}

Keywords: Black Sigatoka, resistance inducers, jasmonic acid, salicylic acid, carophilaceas extract 


\section{INTRODUCCIÓN}

Actualmente en Ecuador se reportan 161583 ha de banano (Santosh y Tiwari 2019), con un total de 356825216 cajas exportadas, representando para el país una importante fuente de ingresos y divisas (Saltos et al., 2020). Esto significa, una generación de alrededor 2,5 millones de empleos a personas que se benefician directa e indirectamente en nueve provincias productoras (Briones et al., 2019; Harari 2004).

La cantidad de fruta producida hace que el país se ubique como el principal exportador en el mundo, dado que el $30 \%$ del banano normalmente comercializado proviene de Ecuador. Esto hace que sea el segundo rubro de mayor importancia económica, manteniéndose hasta la fecha por la elevada cantidad de consumidores a nivel mundial (Briones et al., 2019).

No obstante, a pesar de la cantidad de fruta producida, aún se reportan bajos rendimientos en comparación con nuestros principales competidores en Centro y Sudamérica. Se conoce que uno de los motivos para que la producción se vea afectada, es la enfermedad denominada sigatoka negra, causada por el hongo ascomiceto Mycosphaerella fijiensis Morelet, que, desde su ingreso al país en 1987, ha causado grandes pérdidas anuales (Regalado et al., 2019). Aunado a esto, el manejo de la enfermedad se ha tornado cada vez más costoso y difícil de realizar, principalmente debido a que el patógeno tiene la capacidad de desarrollar resistencia a los principales fungicidas utilizados, particularmente los sistémicos (Munir et al., 2020).

Por estas consideraciones, las investigaciones están siendo orientadas, entre otros aspectos, a conocer el efecto de moléculas inductoras de resistencia a la sigatoka negra, del banano. La inducción de resistencia sistémica adquirida (SAR) o resistencia sistémica inducida (ISR), por sus siglas en inglés, en plantas es un concepto ampliamente distribuido con numerosos estudios que avalan su rol como promotoras de resistencia (Cedeño et al., 2017; Mogollón y Castaño, 2011).

En este contexto, se conoce que en las plantas superiores se pueden activar diversos mecanismos de defensa cuando se exponen a diferentes agentes patógenos, como hongos, bacterias, virus, insectos, etc. Estos mecanismos no permiten el desarrollo de la infección del patógeno, cuando la reacción se produce de manera oportuna. Sin embargo, estos procesos ocurren demasiado tarde o se suprimen, la infección del patógeno se lleva a cabo con éxito, lo que conduce al desarrollo de la enfermedad en la planta (Fullerton y Casonato, 2019).
En este contexto, existen tres hormonas que son clave en lo que concierne a la defensa de plantas: ácido salicílico (SA), jasmonato (JA) y etileno (ET) (Hernandez et al., 2019). SA desencadena principalmente la defensa de la planta contra biotróficos o hemibiotróficos, JA contra los patógenos necrotróficos, aunque hay algunas excepciones. SA actúa como uno de los inductores de resistencia sistémica adquirida en la hoja (Patiño et al., 2010), mientras que JA y ET regulan principalmente la resistencia sistémica inducida desencadenada por PGPR (rizobacterias que promueven el crecimiento de las plantas) (Laredo et al., 2017).

SAR se designa como resistencia mediada por el patógeno necrotizante dependiente del ácido salicílico (SA) para la expresión de proteínas relacionadas con la patogénesis (proteínas $\mathrm{PR}$ ) que desempeña un papel defensivo directo contra los agentes patógenos. SAR difiere de ISR por muchas razones, aunque hay algunas similitudes. Un manejo de la enfermedad en plantas es posible a través de la investigación adecuada de las moléculas de señalización y las moléculas inductoras para la inmunización de las plantas con el fin de inducir la resistencia (Pérez et al., 2009).

La resistencia natural de los vegetales a organismos patógenos es básicamente el efecto combinado de barreras preformadas y mecanismos inducibles en las plantas (Laredo et al., 2017). La utilización indiscriminada y contaminante de fungicidas sintéticos utilizados para el control de sigatoka negra, así como la pérdida de sensibilidad del patógeno hacia las principales moléculas utilizadas para su control, amerita realizar esfuerzos por encontrar productos, que permitan inducir factores de resistencia que contribuyan al desarrollo de una resistencia fisiológica adquirida de las plantas hacia esta y otras enfermedades (Sepúlveda 2016).

Por otro lado, las plantas son organismos, que están obligados a discriminar entre los diferentes retos que les plantea su entorno y responder a ellos. Estas respuestas a su ambiente biótico y abiótico les permiten una mejor distribución de sus recursos para crecer, reproducirse y defenderse, por la presencia de sustancias que son las encargadas de evitar el progreso de la enfermedad (Trinidad-Cruz et al., 2019).

De igual manera una buena nutrición de la planta le brinda las herramientas necesarias para que pueda responder frente al ataque de un patógeno (Vite Cevallos et al., 2020). En base en estos antecedentes, se planteó evaluar a nivel de campo, el efecto de sustancias inductoras de resistencia frente a la evolución de la enfermedad de la sigatoka negra en época seca y lluviosa; esperando inducir los mecanismos de defensa de la planta de banano frente a este problema fitosanitario, así como también sus variables productivas. 


\section{MATERIALES Y MÉTODOS}

El experimento se realizó en un lote experimental localizado en la Estación Experimental Tropical Pichilingue del Instituto Nacional de Investigaciones Agropecuarias (INIAP), ubicada en el km 5 vía Quevedo - El Empalme. Se utilizaron vitroplantas de banano cv. Williams de ocho semanas de edad, las cuales se sembraron en campo, en una superficie de 10707 metros cuadrados, a una altura promedio de $75 \mathrm{msnm}$, temperatura media de $25^{\circ} \mathrm{C}$, humedad relativa de $87 \%$ y una heliófila de 913,73 horas/año. Las coordenadas geográficas son $1^{\circ} 06$ Latitud Sur y $79^{\circ} 25$ de Longitud Occidental.

El cultivo recibió un adecuado y oportuno manejo agronómico, consistiendo en labores de deshije, cirugías fitosanitarias con una frecuencia semanal, fertilización con base en las recomendaciones del laboratorio de manejo de suelos y aguas de la EET-Pichilingue iniciándose 8 semanas después de la siembra. El control de malezas se realizó en forma manual, mecánica y química. Se implementó también riego, en la época seca con una frecuencia de siete días.

Los tratamientos estudiados fueron T1: ácido jasmónico (166 cc), T2: ácido salicílico $(250 \mathrm{cc}), \mathrm{T} 3$ : extracto de carophiláceas (166 cc), más testigos: químico (Aplicaciones de fungicidas siguiendo las recomendaciones de la FRAC) (T4) y absoluto (T5). Las aplicaciones de los tratamientos se realizaron vía foliar a una frecuencia de 10 días durante la época lluviosa (primer ciclo) y de 20 días en la época seca (segundo ciclo), utilizando una aspersora de presión constante (CP3) de 20 litros mientras las plantas de banano estaban aún pequeñas $(100 \mathrm{~cm})$. Cuando las plantas alcanzaron una altura considerable se realizó la aplicación con una aspersora a motor con capacidad de 14 litros. Las aplicaciones se realizaron en horas de la mañana, mientras las condiciones ambientales fueron las adecuadas, es decir sin presencia de lluvias y/o vientos fuertes.

El diseño estadístico utilizado fue Bloques Completos al Azar (DBCA), con 5 tratamientos y cuatro repeticiones cada uno. Cada parcela experimental estuvo conformada por 28 plantas, considerándose únicamente las 10 plantas centrales para el registro de los datos experimentales. Para la comparación de medias entre tratamientos se utilizó la prueba de Tukey al $95 \%$ de confianza.

\section{Variables sanitarias}

Índice y promedio ponderado de infección de sigatoka negra a la floración y cosecha: Este parámetro se evaluó mediante la utilización de la escala de Stover modificada por Gauhl (1989); Mulder y Stover (1976), donde es necesario contar con un patrón o modelo que divide la hoja en proporciones porcentuales, permitiendo así determinar el indice de infección, en cada uno de los tratamientos en estudio.

Hojas funcionales: Esta variable se registró utilizando la escala de Stover modificada por Gauhl (1989); Mulder y Stover (1976). Para su efecto, se consideró como hojas funcionales a aquellas que presentaron hasta el grado 3 de la referida escala.

\section{Variables agronómicas}

Área foliar funcional a la floración y cosecha: En este caso se utilizó el método del nuevo factor propuesto por Kumar et al. (2002), que consiste en multiplicar el largo por el ancho de la tercera hoja por la constante 0,80 por el número de hojas y el factor 0,662. La medición del área foliar se realizó con una cinta graduada en centímetros midiendo el largo y ancho de la lámina foliar.

Peso del racimo (kg): Esta variable se registró en la cosecha, tomando el peso neto de racimo. Se consideró únicamente los frutos que cumplían con las normas para la exportación.

\section{RESULTADOS Y DISCUSIÓN}

El análisis de varianza demostró diferencias estadísticamente significativas entre los tratamientos para la variable índice de infección de sigatoka negra durante la época lluviosa, mientras que en la época seca no se encontró diferencias.

En la figura 1, se puede observar que el testigo químico $(8,39)$ sobresalió en comparación a los demás tratamientos. A. salicílico y A. jasmónico se comportaron de manera similar con índices de infección de 11,38 y 11,60 respectivamente, diferenciándose de $E$. de carophilaceas $(13,75)$. Es conocido que A. salicílico y A. jasmónico, poseen un efecto directo sobre Mycosphaerella fijiensis, tal como lo demuestra Mogollón y Castaño (2011), quienes realizaron evaluaciones a nivel de plántulas de musáceas, encontrando un efecto sobre el hongo, en valores de incidencia y severidad. Por otro lado, Sánchez et al., (2013) manifestaron también que el ácido salicílico tiene un claro efecto sobre los mecanismos de resistencia en plantas. De igual manera Navarro (2019) observó una reducción de la incidencia de patógenos fungosos, por la activación del ácido jasmónico en condiciones de campo, en cultivos de ciclo corto. Finalmente, todos los inductores de resistencia presentaron un menor valor respecto al alcanzado por el testigo absoluto, que fue de 15,62 .

Es importante mencionar, que el T. químico, superó a los demás tratamientos, justificando esto, del por qué, actualmente, es el modo de control convencional de la enfermedad en el cultivo de banano Sepúlveda (2016). 


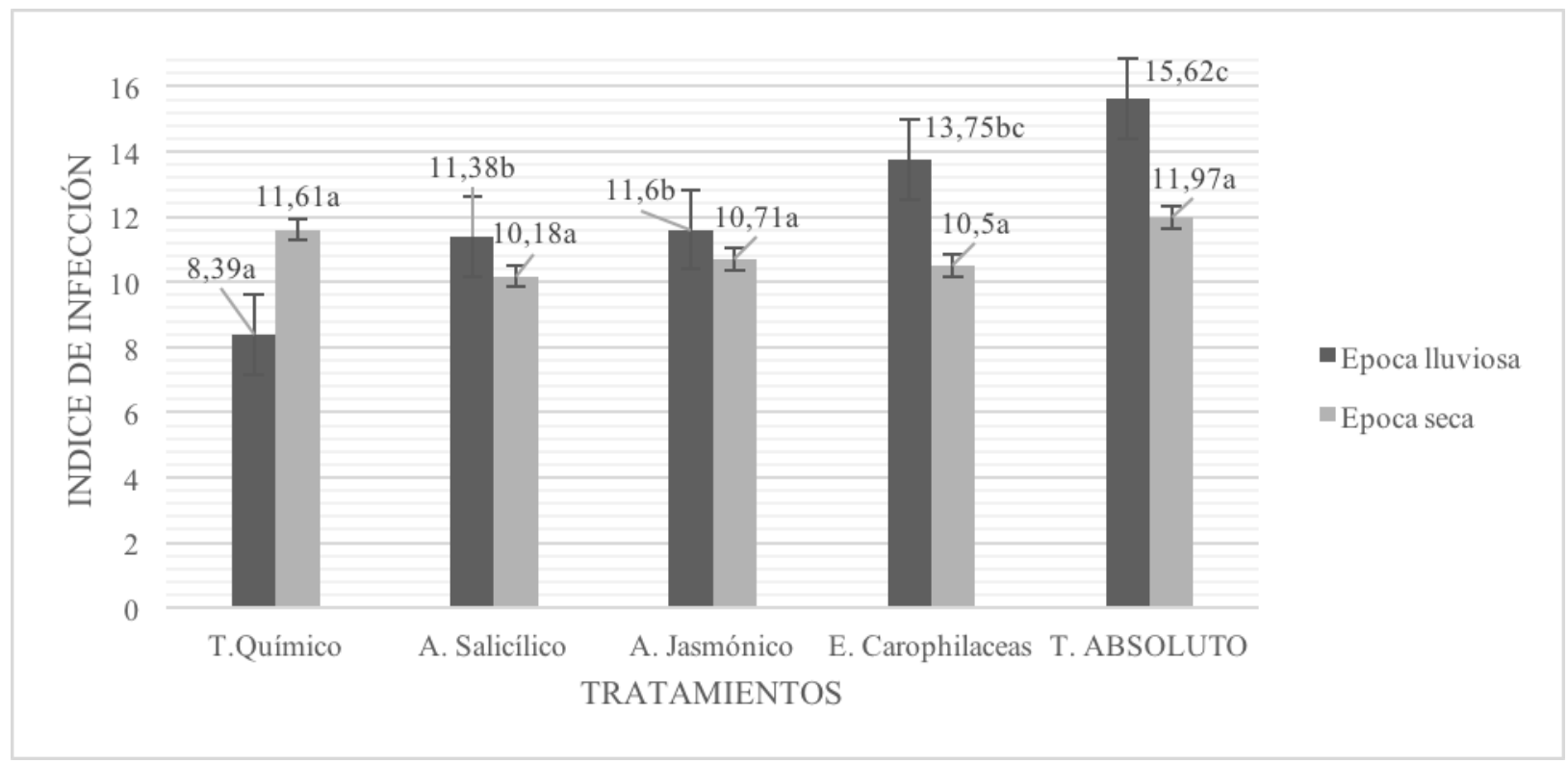

Figura 1. Índice de infección de sigatoka negra, a la floración.

En la figura 2, se puede observar que, de acuerdo al análisis de varianza realizado a la variable índice de infección en plantas de banano en la etapa de cosecha, en las dos épocas (lluviosa y seca), los tratamientos se diferenciaron estadísticamente entre sí.
El testigo químico, presentó los menores índices de infección. En época lluviosa su media fue de 26,48 y finalmente en época seca fue de 21,05.

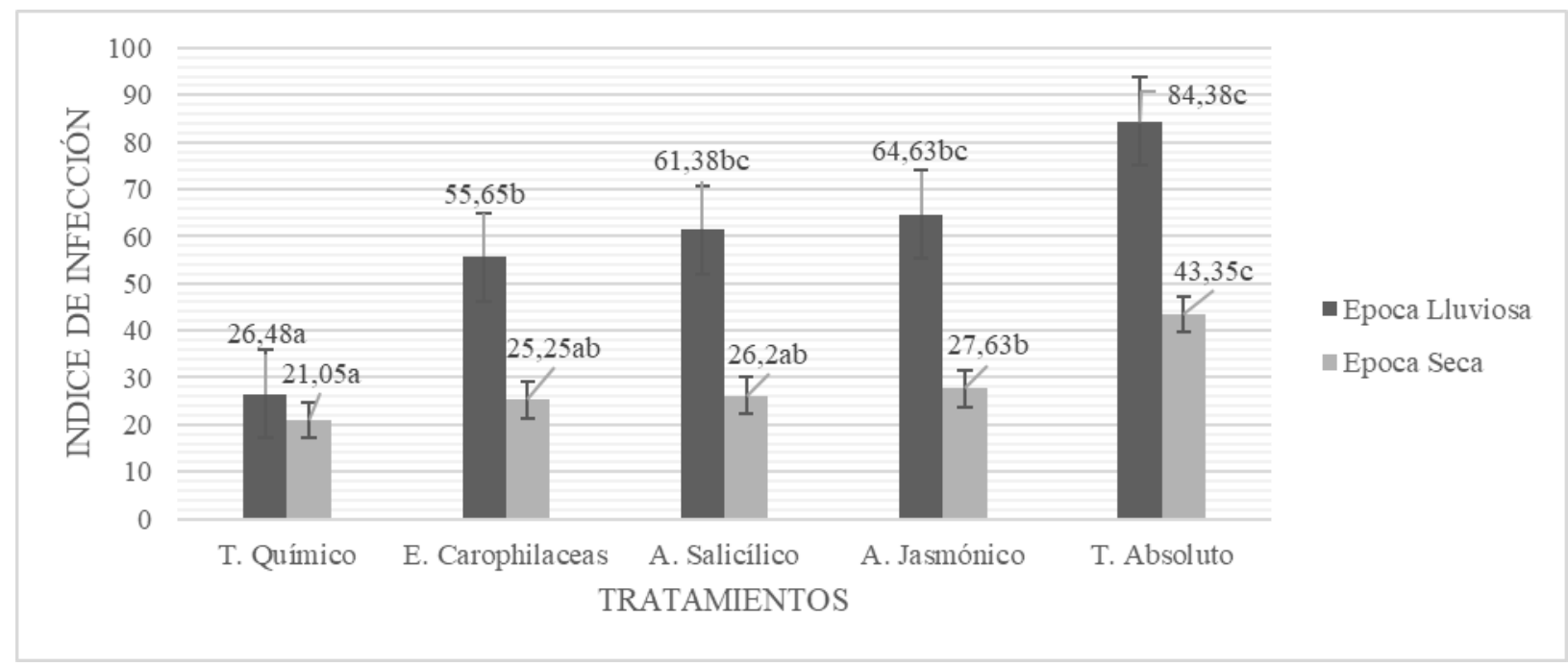

Figura 2. Índice de infección de la sigatoka negra, a la cosecha.

Entre los tratamientos inductores de resistencia, A. salicílico presentó un menor índice de infección, en el primer ciclo de evaluación (época lluviosa), diferenciándose de los demás. Es importante mencionar que al ser un período de lluvias, la presión de inóculo de M. fijiensis, aumenta significativamente (Fullerton y Casonato, 2019; Sepúlveda, 2016). Además, la media registrada por A. salicílico, es relativamente cercano al obtenido por el T. químico, asemejándose a los reportados por Patiño etal. (2010), quien usó comparaciones de inductores de resistencia frente a sustancias de síntesis química en el cultivo de banano. Ya en la época seca, E. de carophiláceas y A. salicílico 
presentaron menores índices frente al tratamiento testigo absoluto y A. jasmónico.

En la figura 3, se puede observar el comportamiento de cada uno de los tratamientos estudiados. El análisis de varianza no reflejó diferencia estadística y no hubo ningún efecto de los tratamientos sobre la variable hojas funcionales. Sin embargo, el E. de carophiláceas presentó, junto con el testigo químico, las mejores medias en la época lluviosa, siendo esto un factor indispensable en el cultivo, es decir, alcanzar un oportuno número de hojas en este momento del ciclo de la planta (Dos Santos et al., 2019). En la época seca, los inductores de resistencia fueron superiores numéricamente, respecto a los testigo absoluto y químico.

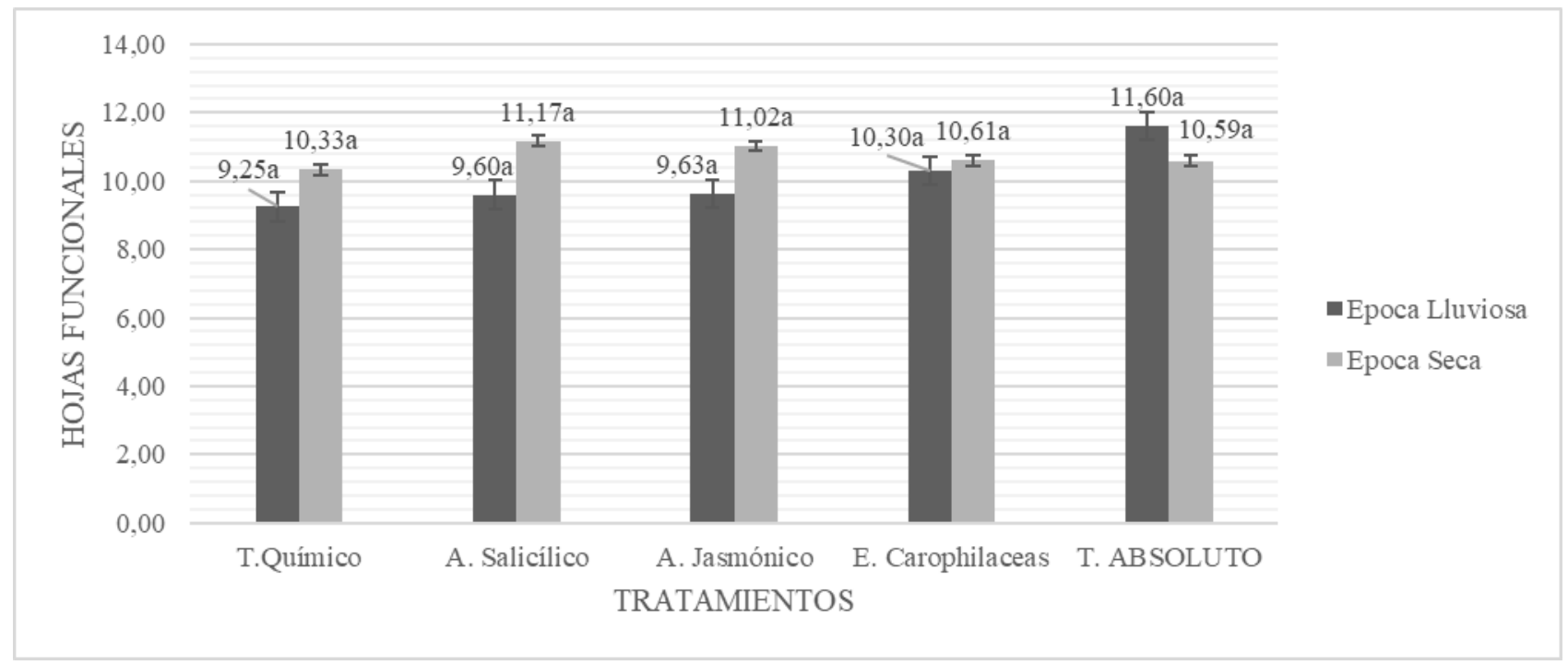

Figura 3. Hojas funcionales en la floración de plantas de banano.

En la figura 4, se puede observar que, de acuerdo al análisis de varianza realizado, existió diferencia estadística, en la época seca y lluviosa. El testigo químico fue el mejor tratamiento, con mayor número de hojas funcionales a la cosecha, en cada época. En lo que corresponde a los inductores de resistencia, el E. de carophiláceas $(1,13)$ presentó un promedio sobresaliente respecto a los demás inductores, incluyendo el testigo absoluto $(0,25)$ durante la época lluviosa.

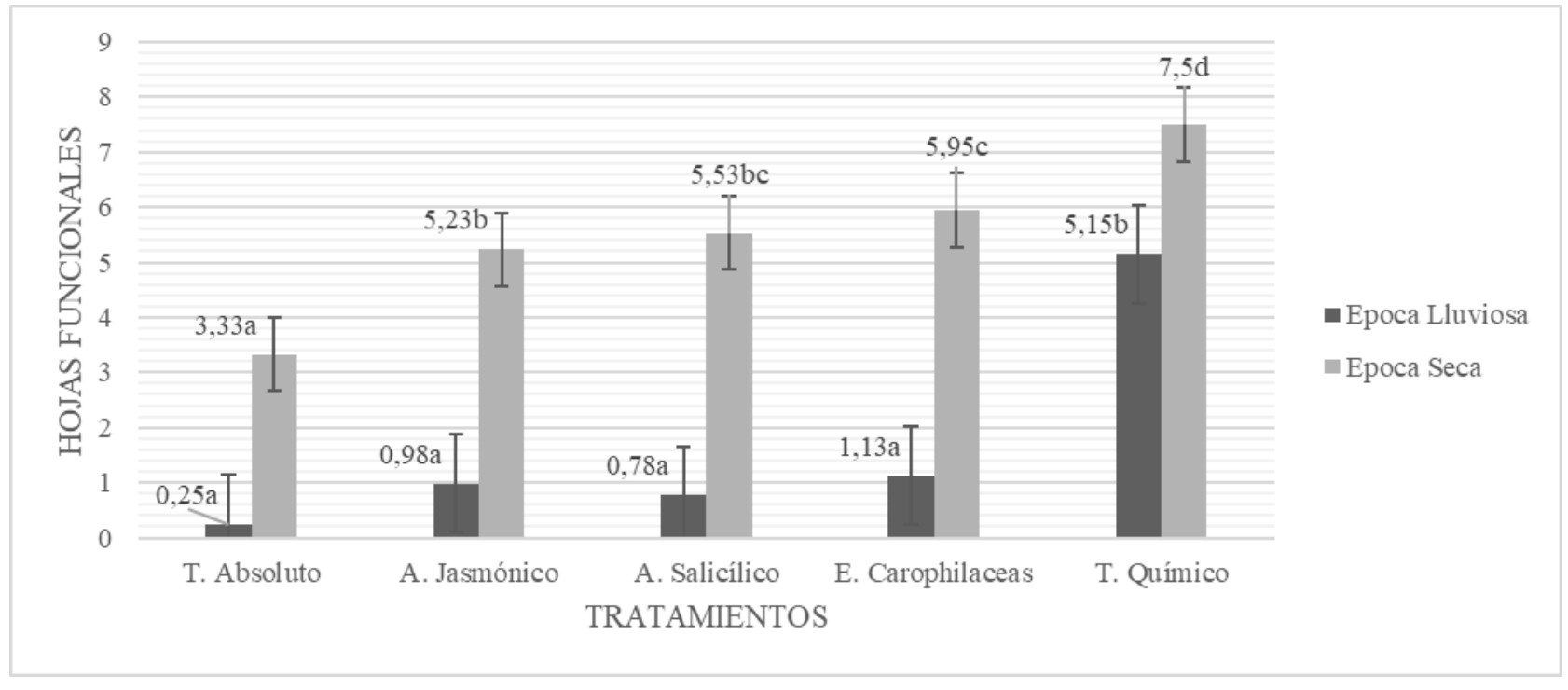

Figura 4. Hojas funcionales en la etapa de cosecha 
Cabe recalcar que durante la época seca existió diferencia estadística, resaltando entre los inductores de resistencia nuevamente el E. de carophiláceas $(5,95)$ como el mejor, seguido por el A. salicílico $(5,53)$. Todos los inductores fueron superiores al testigo absoluto, que únicamente alcanzó un promedio de 3,33 hojas funcionales, estos datos fueron similares a los obtenidos por Sánchez y
Stalin (2017) donde al evaluar sustancias bioestimulantes, a base de algas, reportaron efectos sobre la sigatoka negra en banano.

En la figura 5, se puede observar que los tratamientos no fueron diferentes en la variable área foliar. Comportándose en el primero como en el segundo ciclo de forma similar.

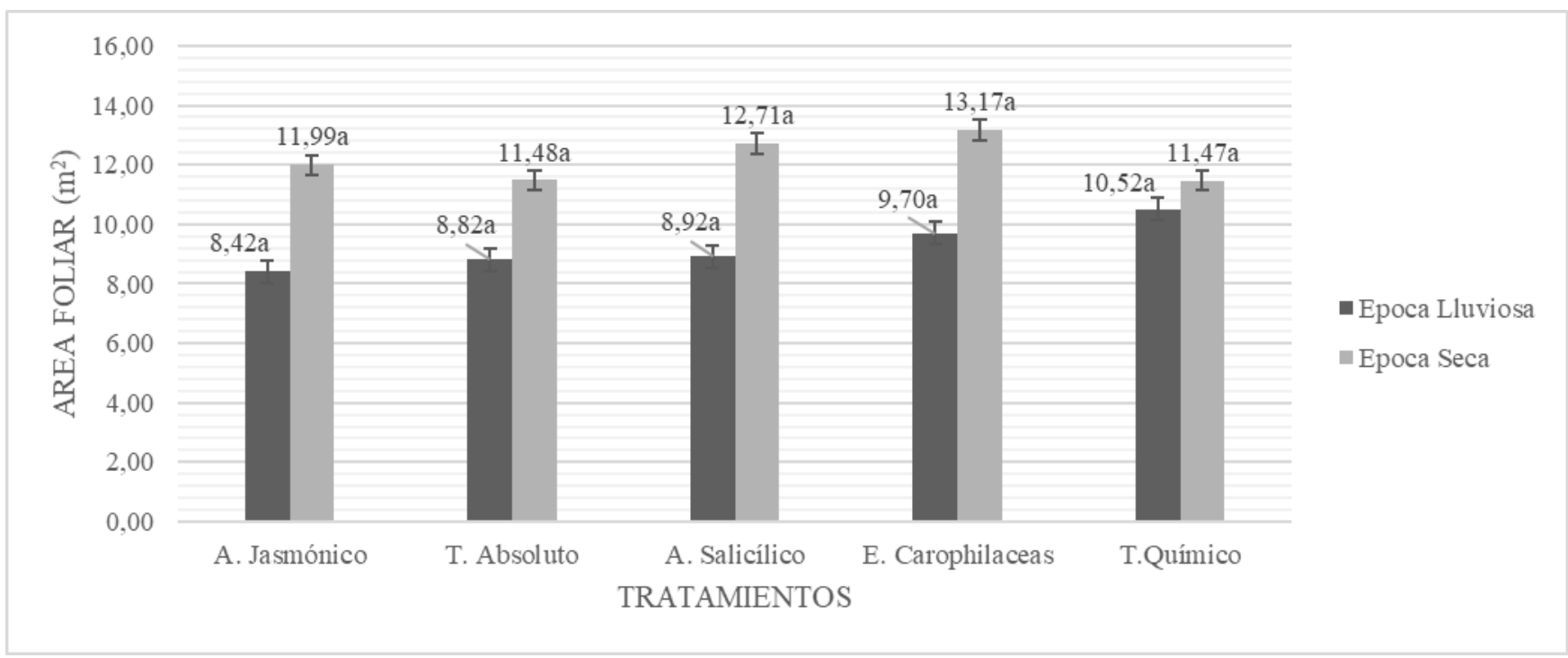

Figura 5. Área foliar evaluada durante la etapa a la floración

En la figura 6, se observa diferencia estadística entre los tratamientos en estudio. En el primer ciclo (época lluviosa), el testigo químico, fue superior a los demás, con un promedio de 4,7. Los inductores de resistencia fueron iguales estadísticamente, siendo únicamente el E. de carophiláceas $(1,1)$ el que se acercó al valor obtenido por el testigo químico.

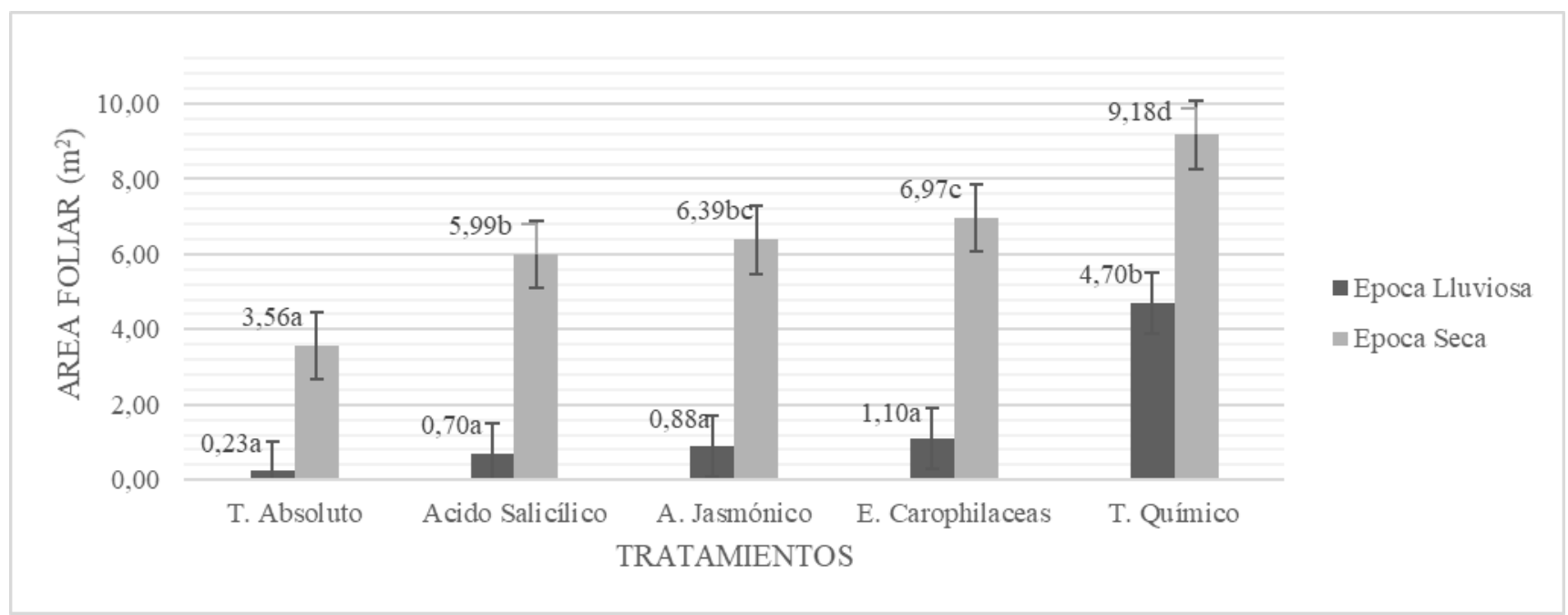

Figura 6. Área foliar evaluada durante la cosecha 
En el segundo ciclo (época seca), se observa diferencia estadística en todos los tratamientos estudiados. El testigo químico, resultó ser el mejor, con un promedio de 9,18 . En cuanto a los inductores de resistencia todos fueron superiores al testigo absoluto $(3,56)$. El E. de carophiláceas fue el mejor de los inductores evaluados. Fullerton y Casonato (2019) mencionan que al hacer evaluaciones similares con el estado asexual del agente causal de la sigatoka negra, demostraron un efecto directo sobre el hongo, así también Sepúlveda (2016) reportó una sensibilidad del hongo a sustancias provenientes de algas.
Respecto a la variable peso neto del racimo, en el primer ciclo (época lluviosa) no se observó diferencia estadística en ninguno de los tratamientos evaluados (Figura 7). En el segundo ciclo, A. salicílico fue diferente a todos los inductores con un promedio de 31,93 y a los testigos químico y absoluto. Se conoce que este ácido, influye sobre las plantas en sus mecanismos de defensa (Patiño et al., 2010: Sánchez et al., 2013). Además, dentro de estos, se ha comprobado que al indicador reacciones de defensa en la planta, influye también en comportamiento agronómico de los vegetales a nivel de producción (Hernández et al., 2019).

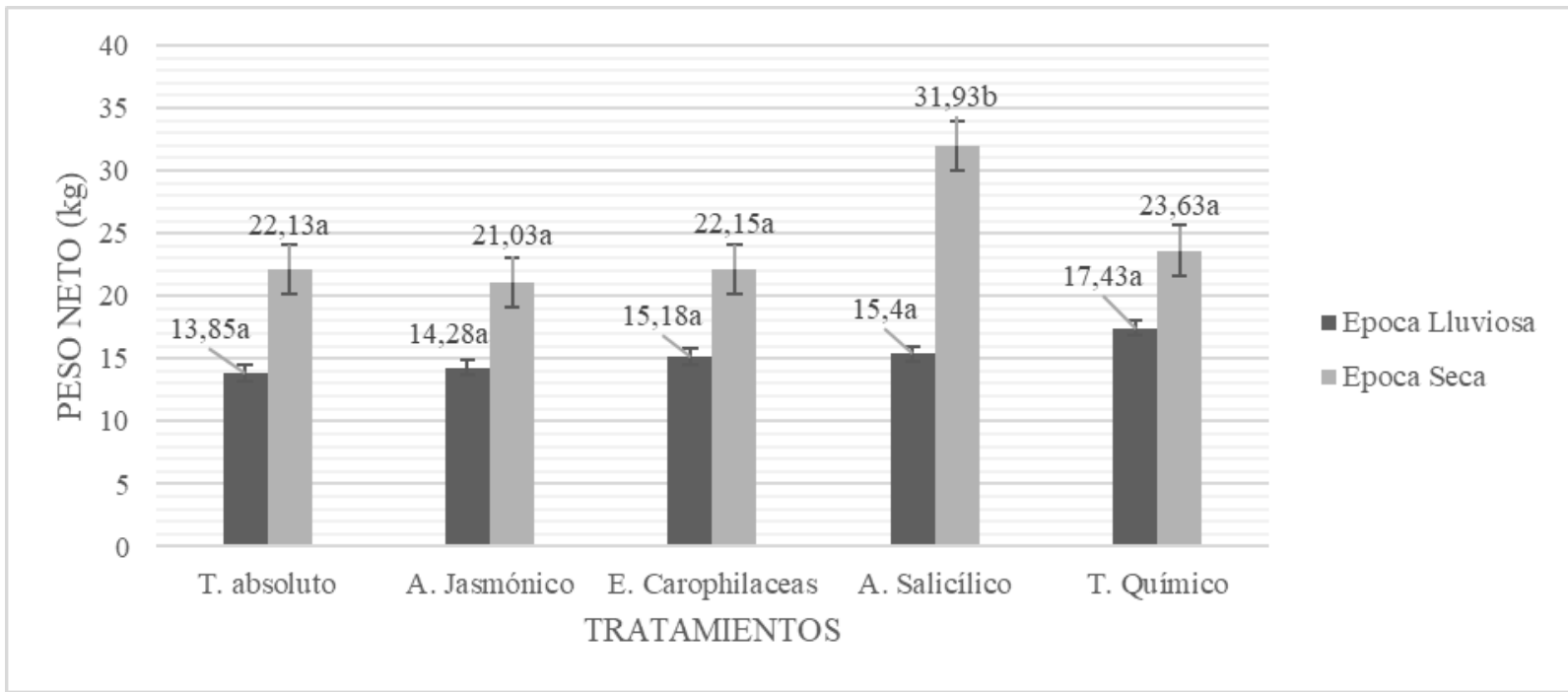

Figura 7. Peso neto del racimo registrado en época lluviosa y seca

\section{CONCLUSIONES}

Los inductores de resistencia mostraron un efecto sobre la sigatoka negra en comparación con el testigo absoluto, comportándose mejor los tratamientos ácido salicílico y extracto de carophiláceas.

Acido salicílico en epoca seca, presentó mejor promedio en cuanto a peso neto, valores superiores a los otros tratamientos.

\section{LITERATURA CITADA}

Briones, J., Novillo, Lenin A., y Hans, E. 2019. Apreciación del dólar en las exportaciones de banano ecuatoriano, hacia el mercado Ruso. Revista Científica Ciencia y tecnología 19(22).

Cedeño, G., Suarez, D., Vera, C., Devra, J., y Santis. P. 2017. Detección temprana de resistencia a
Mycosphaerella fijiensis en genotipos locales de Musáceas en Ecuador». Scientia Agropecuaria 8(1):29-42.

Dos Santos Felix, Evaldo, Daniel Duarte Pereira, George Vieira do Nascimento, Elder Cunha de Lira, Tarcisio José de Oliveira Filho, Jaqueline de Araújo Oliveira Machado, y Cleomara Gomes de Sousa. 2019. «Diagnóstico do cultivo da banana em uma região do Brejo Paraibano/Diagnosis of banana production in a region of Brejo Paraibano»». Brazilian Journal of Development 5(12):29616-32.

Fullerton, R. A. y S. G. Casonato. 2019. The infection of the fruit of 'Cavendish' banana by Pseudocercospora fijiensis, cause of black leaf streak (black Sigatoka)». European Journal of Plant Pathology 155(3):779-87.

Gauhl, F. 1989. Epidemiology and ecology of Black Sigatoka on plantain and banana (Musa spp.) in 


\section{Costa Rica, Centroamérica».}

Harari, Raúl. 2004. Efectos sociales de la globalización: petróleo, banano y flores en Ecuador. Editorial Abya Yala.

Hernandez, D., Ayala, D., Ruiz Sáenz, E., Cruz Gutiérrez, y López., H. 2019. Ácido salicílico induce tolerancia al estrés por criogenia en Solanum tuberosum». Revista mexicana de ciencias agrícolas 10(7):1505-15.

Kumar, N., V. Krishnamoorthy, L. Nalina, y K. Soorianathasundharam. 2002. «A new factor for estimating total leaf area in banana».

Laredo Alcalá, E., Iñaky, José L. Martínez, A, Guillen., L, y Hernández, F. 2017. «Aplicación de ácido jasmónico como inductor de resistencia vegetal frente a patógenos»». Revista mexicana de ciencias agrícolas 8(3):673-83.

Mogollón Ortiz, Ángela y Jairo Castaño Zapata. 2011. Efecto de inductores de resistencia en plántulas de plátano dominico-hartón (musa balbisiana aab) contra Mycosphaerella spp.. Revista de la Academia Colombiana de Ciencias Exactas, Físicas y Naturales 35(137):463-71.

Mulder, J. L. y R. H. Stover. 1976. «Mycosphaerella species causing banana leaf spot.» Transactions of the British Mycological Society 67(1):77-82.

Munir, Misbakhul, Hehe Wang, Paula Agudelo, y Daniel J. Anco. 2020. «Rapid Detection of Fungicide Resistance Phenotypes Among Populations of Nothopassalora personata in South Carolina Peanut Fields». Plant Health Progress 21(2):12332.

Navarro, L. T. 2019. «Control biológico de botrytis por la cepa Bacillus sp. e implicaciones en la producción de plantas de interés agrícola».

Patiño H., Vasquez D. LE, Aristizabal G. MI, Castañeda S. DA, y Mira C. JJ. 2010. «Acido salicilico y acibenzolar-s metil como inductores de resistencia en el control de sigatoka negra». ACORBAT, 19, 2010, Medellin.

Pérez Vicente, Luis, Alicia Batlle Viera, Julio Chacón Benazet, y Virgen Montenegro Moracén. 2009. «Eficacia de Trichoderma harzianum a34 en el biocontrol de Fusarium oxysporum f. Sp. cubense, agente causal de la marchitez por fusarium o mal de Panamá de los bananos en Cuba». Fitosanidad 13(4):259-64.
Regalado, J. G., Augusto, P., y Palacios, C. 2019. "Amenazas de las manchas foliares de Sigatoka, Mycosphaerella spp., en la producción sostenible de banano en el Ecuador». Revista Verde de Agroecologia e Desenvolvimento Sustentável 14(5):591-96.

Saltos, Amparito Cecilia León, Juan Gabriel Saltos Cruz, y Estefanía de los Ángeles Villagrán Guerrero. 2020. «Estado actual de los procesos de comercialización del sector bananero en la provincia de los Ríos, Ecuador». Universidad Ciencia y Tecnología 24(99):11-16.

Sánchez, Cedeño y Eddy Stalin. 2017. «Efectos de estimulantes orgánicos y fertilización potásica sobre la resistencia a Sigatoka Negra (Mycosphaerella fijiensis) y producción en el cultivo de banano (Musa paradisiaca) en el cantón Buena Fe».

Sánchez, Gerardo Rangel, Elda Castro Mercado, Elda Beltran Peña, Homero Reyes de la Cruz, y Ernesto García Pineda. 2013. «El ácido salicílico y su participación en la resistencia a patógenos en plantas»». Revista de la DES Ciencias Biológico Agropecuarias. Universidad Michoacana de San Nicolás de Hidalgo 12:90-95.

Santosh, D. T. y K. N. Tiwari. 2019. «Estimation of water requirement of Banana crop under drip irrigation with and without plastic mulch using dual crop coefficient approach». P. 12024 en IOP Conference Series: Earth and Environmental Science. Vol. 344. IOP Publishing.

Sepúlveda, Leonardo. 2016. «Caracterización fenotípica de Mycosphaerella fijiensis y su relación con la sensibilidad a fungicidas en Colombia». Revista mexicana de fitopatología 34(1):1-21.

Trinidad-Cruz, J., Rincón-Enríquez., G., QuiñonesAguilar., E., Arce-Leal., A. Leyva-López., N. 2019. "Inductors of plant resistance in the control of Candidatus Liberibacter asiaticus in Mexican lemon (Citrus aurantifolia) trees.» Revista Mexicana de Fitopatología 37(2).

Vite Cevallos, Harry, Héctor Carvajal Romero, y Salomón Barrezueta Unda. 2020. «Aplicación de algoritmos de aprendizaje automático para clasificar la fertilidad de un suelo bananero»». Conrado 16(72):15-19. 